\title{
Design and Simulation of MC-CDMA Transceiver via Slantlet Transform
}

\author{
Kattoush A. H. \\ Electrical Engineering Department, Tafila Technical University, Jordan, \\ Email:akattoush@yahoo.ca \\ Qasaymeh M. M. \\ Electrical Engineering Department, Tafila Technical University, Jordan, \\ Email: qasaymeh@ttu.edu.jo
}

\begin{abstract}
Wireless communications is a rapidly growing piece of the communications manufacturing, with the potential to provide high-speed high-quality information exchange between the portable devices located anywhere in the world. Multi-Carrier Code Division Multiple Access (MC-CDMA) has emerged recently as a promising candidate for the next generation broad-band mobile networks. Recently, it was found that Slantlet transform (SLT) based Orthogonal Frequency Division Multiplexing (OFDM) is capable to reduce the Inter Symbol Interference (ISI) and the Inter Carrier Interference (ICI), which are caused by the loss of orthogonality between the subcarriers. SLT-OFDM can support higher spectrum efficiency than Fast Fourier Transform-based OFDM (FFT-OFDM) due to the elimination of the Cyclic Prefix (CP). In this paper, a novel SLT-MC-CDMA transceiver design is presented based on the SLT-OFDM that is used as a basic building block in the design of MC-CDMA transceiver to maintain the orthogonality against the multipath frequency Selective Fading Channels (SFC). Simulation results are provided to demonstrate the significant gain in the performance of the proposed technique. The Bit Error Rate (BER) of SLT-MC-CDMA scheme is compared with FFT-MC-CDMA and tested in Additive White Gaussian Noise (AWGN), Flat Fading and Selective Fading Channels (SFH). The simulation results confirmed that, the proposed system outperforms the reference one.
\end{abstract}

Index Terms-Slantlet Transform; Slantlet based OFDM; MC-CDMA; Selective Fading Channel.

\section{Introduction}

Wireless communications ${ }^{[1-4]}$ is a rapidly growing piece of the communications manufacturing, with the potential to provide high-speed high-quality information exchange between the portable devices located anywhere in the world. Multi-path fading channels have a severe effect on the performance of wireless communication systems, even those systems that exhibit efficient bandwidth, like Orthogonal Frequency Division Multiplexing (OFDM) and Multi-Carrier Code Division Multiple Access (MCCDMA). There is always a need for developments in the realization of these systems as well as efficient channel estimation and equalization methods to enable these systems to reach their maximum performance ${ }^{[1]}$.

CDMA has been a robust candidate to support multimedia mobile services because it has the capability to handle with the asynchronous nature of the multimedia traffic and it can afford higher capacity as opposed to the conventional access schemes ${ }^{[2,3]}$.

The main problem in the design of a communications system over a wireless link is to deal with multi-path fading, which causes a significant degradation in terms of both the reliability of the link and the data rate ${ }^{[4]}$.

Recently, a number of new MC-CDMA systems have been proposed as an alternative to the classical single-carrier CDMA systems ${ }^{[5-10]}$. The principle of CDMA was combined with OFDM, which allows one to use the available spectrum in an efficient way and maintain the many advantages of a CDMA system ${ }^{[1]}$. This combination of OFDM-CDMA or MC-CDMA was first proposed in ${ }^{[11]}$. It is a useful technique for 4-th generation (4G) systems where variable data rates as well as reliable communication systems are required. Combining OFDM with CDMA has one key advantage; it can lower the symbol rate in each subcarrier compared to OFDM so that longer symbol duration makes it easier to synchronize. 


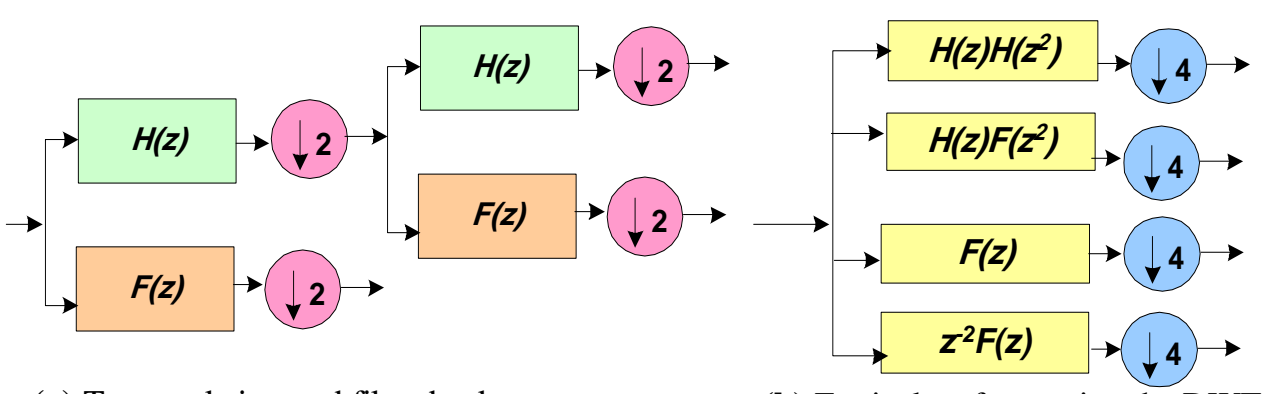

(a) Two-scale iterated filter-bank

(b) Equivalent form using the DWT

Fig. 1: Two-scale iterated filter-bank and its equivalent form using the DWT.

MC-CDMA not only mitigates the ISI but also exploits the multipath ${ }^{[12]}$. The same idea was proposed again and the performance of maximum likelihood detection for MC-CDMA systems was analyzed ${ }^{[13]}$. The Fourier based complex exponential carriers of the OFDM system was interchanged with some orthonormal wavelets in order to reduce the level of interference. The wavelets are derived from multistage tree-structured Haar and Daubechies orthonormal Quadrature Mirror Filter bank. Comparing with the conventional OFDM, it was found that the Haar and Daubechies-based orthonormal wavelets are capable of reducing the power of ISI and ICI ${ }^{[14]}$.

The FFT-OFDM has currently drawn most of attention in the area of wireless communication. To combat ISI and ICI, Cyclic Prefix (CP) is inserted between FFT-OFDM symbols which take around 25 percent of bandwidth. To improve the bandwidth efficiency and reduce the ISI and ICI Discrete Wavelet Transform (DWT) based OFDM was proposed and studied extensively. A comparison between FFT-OFDM and DWT-OFDM at different transmission scenarios can be found in ${ }^{[15,16]}$.

DWT-based MC-CDMA has lately gained popularity in literature due to very high spectral containment properties of wavelet filters ${ }^{[17-19]}$. Waveletbased MC-CDMA can better combat narrowband interferences and more robust to ICI than traditional FFT filters. Moreover, since the classic notion of a guard band does not apply for DWT-MC-CDMA; data rates can be enhanced over those of FFT implementations.

In order to further reduce the level of interference, increase the spectral efficiency, and reduce the computation complexity FFT is replaced with Slantlet Transform (SLT) in MC-CDMA design in this paper. The performance comparisons of FFT-MC-CDMA and SLT-MC-CDMA on three different channel models: AWGN, flat fading and selective frequency fading is provided. Simulation results show that proposed SLT design achieves much lower Bit Error Rates (BER),increases Signal to Noise power Ratio (SNR), and can be used as an alternative to the conventional MCCDMA.

In this paper, a novel SLT-MC-CDMA transceiver design is presented based on the SLT-OFDM that is used as a basic building block in the design of MC-CDMA transceiver to maintain the orthogonality against the multi-path frequency SFC. The rest of this paper is organized as follows. Section two introduces the Slantlet based OFDM system. Section three presents the proposed realization of SLT-based MC-CDMA. Section four provides the performance analysis of the proposed SLT-MC-CDMA including simulation results to demonstrate the significant gain in the performance of the proposed technique.

\section{Slantlet based OFDM system}

The SLT was first proposed by Selesnick ${ }^{[20]}$. It is an orthogonal DWT with two zero moments and with improved time localization. It uses a special case of basis ${ }^{[20]}$, the construction of which relies on Gram-Schmidt orthogonalization. Selesnick described the basis from a filter-bank viewpoint, gave explicit solutions for the filter coefficients, and described an efficient algorithm for the transform.

The usual iterated DWT filter-bank and its equivalent1 form are shown in Fig. 1. The Slantlet filterbank is based on the equivalent structure that is occupied by different filters that are not products. With this extra degree of freedom obtained by giving up the product form, filters of shorter length are designed satisfying orthogonality and zero moment conditions.

For two-channel case the Daubechies filter ${ }^{[22]}$ is the shortest filter which makes the filter-bank orthogonal and has $K$ zero moments. For $K=2$ zero moments, filters $H(z)$ and $F(z)$ are of length 4 . For this system, the iterated filters in Fig. 1 are of length 10 and 4 . Without the constraint that the filters are products, an 


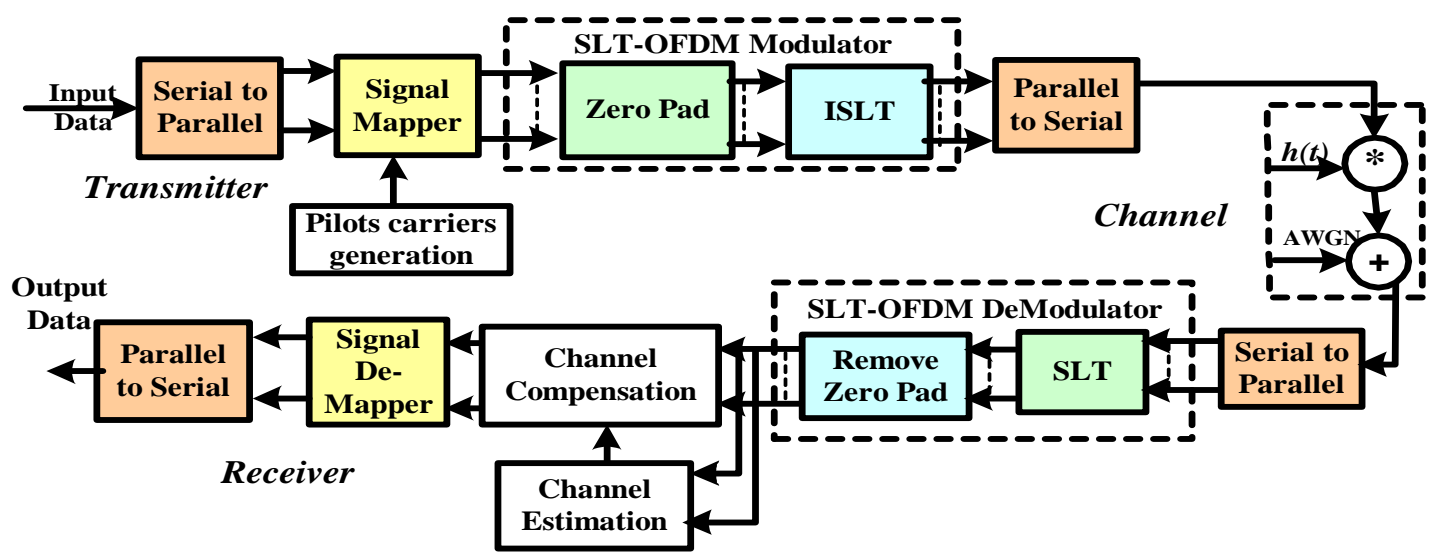

Fig. 2: Block Diagram of SLT-OFDM System

orthogonal filter-bank with $K=2$ zero moments can be obtained where the filter lengths are 8 and 4 . That is a reduction by two samples, which is a difference that grows with the number of stages. This reduction in length, while maintaining desirable orthogonality and moment properties, is possible because these filters are piecewise linear. Even though there is no tree structure for SLT, it can be efficiently implemented like an iterated DWT filter-bank ${ }^{[20]}$. Therefore, a computational complexity of the SLT is of the same order as that of the DWT.

The block diagram of the SLT-OFDM system is depicted in Fig. 2. It is very similar the FFT-OFDM with some differences in the OFDM modulator-demodulator blocks. The processes of serial to parallel (S/P) conversion, signal de-mapping, and training sequence insertion are the same. The SLT-based OFDM modulator consists of zero padding and Inverse SLT (ISLT) blocks while the SLT-based OFDM demodulator consists of SLT and zero-pad removal blocks. The main difference between FFT-OFDM and SLT-OFDM is that in SLTOFDM there is no need for adding a CP to OFDM symbols; therefore the spectral efficiency and the data rate of SLT-OFDM are better than those of FFT-OFDM.

\section{Proposed realization of SLT-based MC- CDMA}

The MC-CDMA transmitter spreads the original data stream over different sub-carriers using a given spreading code in the frequency domain ${ }^{[11,13]}$. Fig. 3 and Fig. 4 show the MC-CDMA transmitter of the $k^{\text {th }}$ user for BPSK scheme and the power spectrum of the transmitted signal respectively ${ }^{[9]}$, where $d_{k}(t)$ is the data bits for $k^{\text {th }}$ user, $G_{M C}$ denotes the processing gain, $N_{C}$ is the number of sub-carriers and $C_{k}(t)$ is the spreading Pseudo-random Noise (PN) sequence of the $k^{\text {th }}$ user. It is assumed that $N_{C}=G_{M C}{ }^{[11]}$. In MC-CDMA systems, the original data stream from a user is spread with the user's specific spreading code in the frequency domain. In other words, a fraction of the symbol corresponding to a chip of the spreading code is transmitted through a different subcarrier. The narrowband subcarriers are generated using BPSK modulated signals, each at different frequencies, which at baseband are multiples of harmonic frequency $1 / T_{s}$. That is,

$$
\Delta f=f_{i}-f_{i-1}=\frac{1}{T_{S}}
$$

where, $T_{s}$ is the symbol duration of data stream. The subcarrier frequencies are orthogonal to each other at baseband. The transmitted signal of the $k^{\text {th }}$ user is given by:

$$
S_{k}^{M C}(t)=d_{k}(t) \sum_{i=1}^{N_{C}} c_{k}^{i} \cos \left(\omega_{i} t\right)
$$

The total bandwidth required for transmission is:

$$
\beta=\frac{\left(N_{C}+1\right) G_{M C}}{N_{C} T_{S}}
$$

The received signal of MC-CDMA systems ${ }^{[9]}$ for all $K$ users as Fig. 5 shows is given by:

$$
r(t)=\sum_{k=1}^{K} d_{k}(t) \sum_{i=1}^{N_{C}} h(i) c_{k}^{i} \cos \omega_{i} t+n(t)
$$




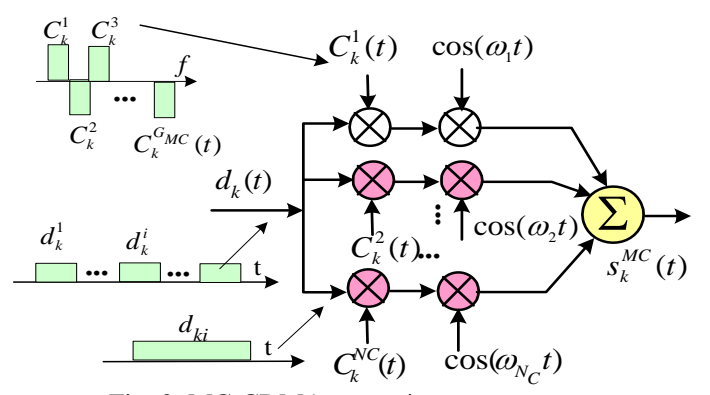

Fig. 3: MC-CDMA transmitter system model

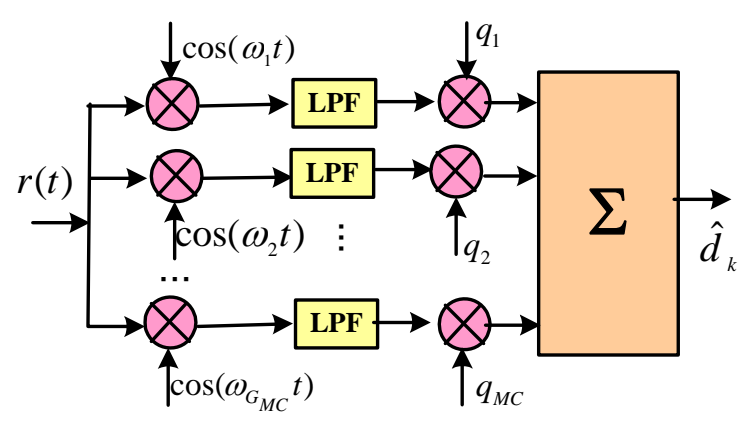

g. 5: MC-CDMA receiver system model.

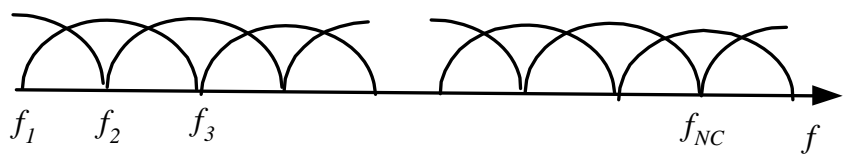

Fig. 4: Power spectrum representation of the transmitted signal.

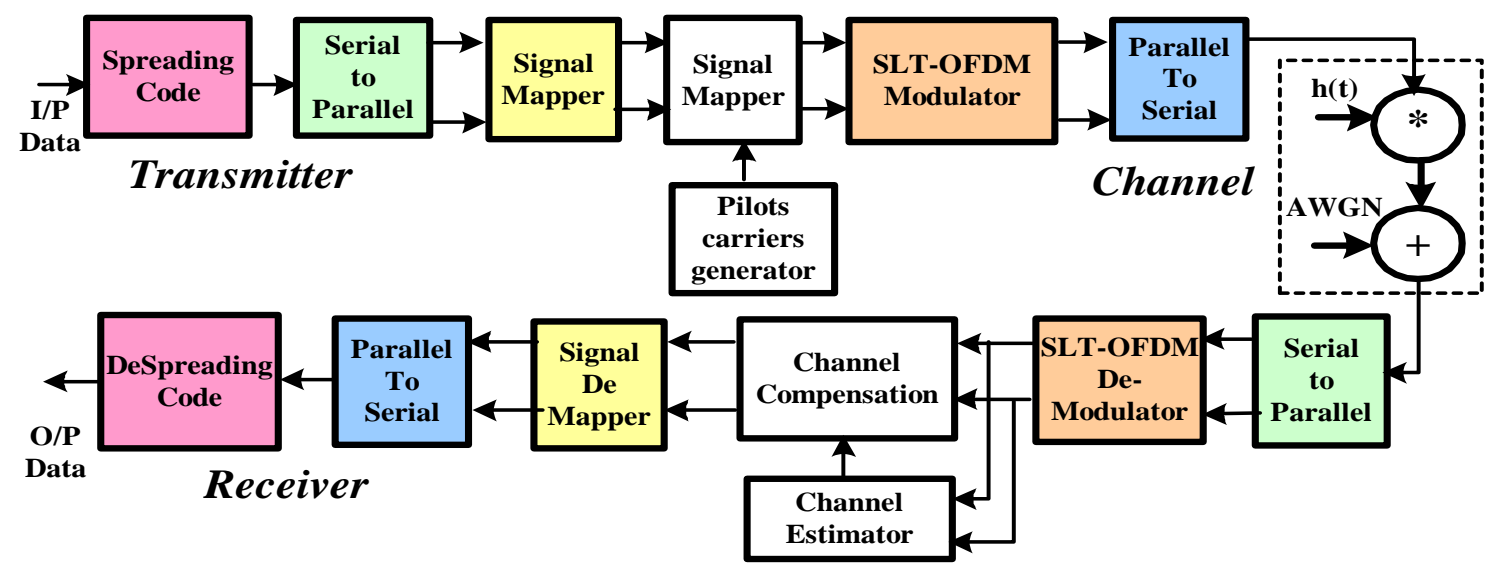

Fig. 6: Block Diagram of proposed SLT-MC-CDMA System

where $n(t)$ is the Additive White Gaussian Noise (AWGN) and $h(i)$ is the complex signal channel coefficient of the $i^{\text {th }}$ subcarrier. The received signal is demodulated with corresponding subcarrier followed by low pass filtering to generate the baseband signal.

The baseband signal is weighted by some coefficients, and then all baseband signals are combined together. It can be seen that the received signal is combined in the frequency domain therefore the receiver can always employ all the received signal energy scattered in the frequency domain. This is the advantage of MC-CDMA $[11,13]$. Different combining techniques to enhance the system performance can be used, which correspond to different choices of coefficients. Typical combining techniques include maximum ratio combining, equal gain combining ${ }^{[11]}$ and minimum mean square error combining ${ }^{[13]}$. The drawback of this system is that, it does not consider the multipath and fading effects.

The block diagram of the proposed system for SLT based MC-CDMA is depicted in Fig. 6. In this design the
FFT-Based OFDM is replaced with a SLT-based OFDM which has a better performance and a reduced ISI and ICI in comparison with FFT-based OFDM. Each data symbol is multiplied with a spreading sequence, the Gold sequence can be used since it has a relatively good correlation values. Other spreading codes that have a relatively good correlation values like Walsh-Hadamard (WH) code can be used, but it can be used when a small number of users are considered, since the orthogonality of the code is reduced due to the multipath propagation [23].

Binary Phase Shift Keying (BPSK) or Quadrature Phase Shift Keying (QPSK) signal mapping can be used for mapping the spreading and the training sequences. After that a pilot-carrier (training sequence) which may be a bipolar sequence previously informed to receiver is generated. The channel frequency response is estimated by using the training and the received sequences as follows: 


$$
H(k)=\frac{\text { received Training Sample }(k)}{\text { transmitte d Training Sample }(k)}
$$

The channel frequency response previously found is used to compensate the channel effects on the data.

\section{Performance Analysis of proposed SLT-MC- CDMA}

In this section the results of BER performance simulations using MATLAB 7 for SLT-MC-CDMA are provided and compared with the conventional FFT- MCCDMA under different channel conditions. AWGN channels, Flat Fading Channels (FFC), and multi-path frequency selective Rayleigh distributed with AWGN channels are considered during simulations. The system parameters used in the simulation are as follows: $5 \mathrm{MHz}$ bandwidth, BPSK modulation, Walsh-Hadamard spreading code, and 64 sub-carriers.

\subsection{Performance of SLT-MC-CDMA in AWGN Channel}

The BER performance of the proposed SLT-MCCDMA and the conventional FFT-MC-CDMA system in AWGN channel is shown in Fig. 7. The SNR is changed from $0 \mathrm{~dB}$ to $40 \mathrm{~dB}$. From which it can be noted that SLTMC-CDMA has an SNR gain of $16 \mathrm{~dB}$ compared with FFT-MC-CDMA system to achieve BER of $10^{-2}$. Also it has an SNR gain of $16 \mathrm{~dB}$ compared with FFT-MCCDMA system to achieve BER of $10^{-3}$.

\subsection{Performance of SLT-MC-CDMA in a Flat Fading Channel}

The BER performance of SLT-based MC-CDMA in a Flat Fading Channel is tested under a two-ray Rayleighdistributed multi-path fading channel model is assumed. The second path gain and delay was set to $-8 \mathrm{~dB}$ and $1 \mu \mathrm{sec}$ respectively. It is assumed that all frequency components of the transmitted signal are changed and correlated in phase and magnitude.

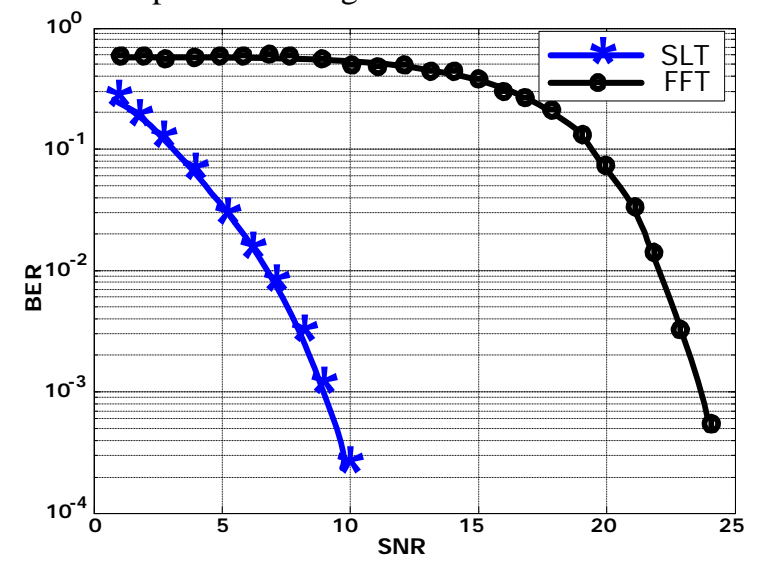

Fig. 7: Performance of MC-CDMA in AWGN channel.

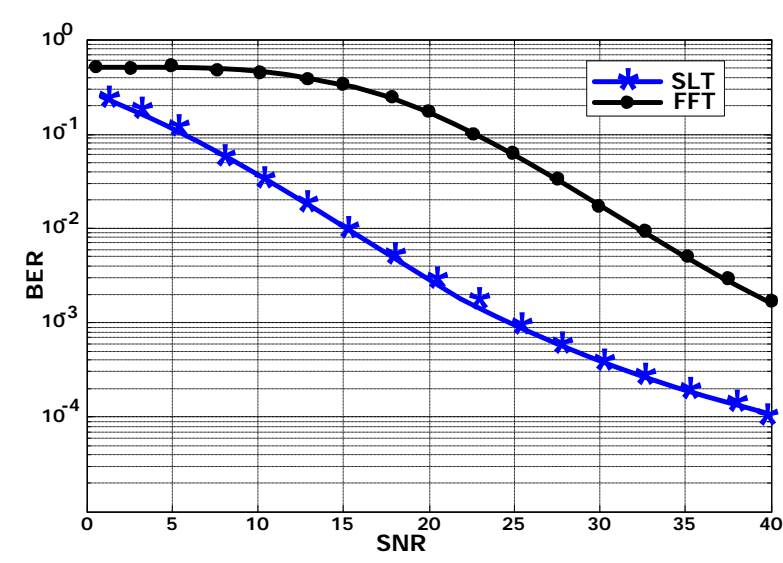

Fig. 8: Performance of SLT-MC-CDMA in FFC at maximum Doppler shift $=5 \mathrm{~Hz}$.

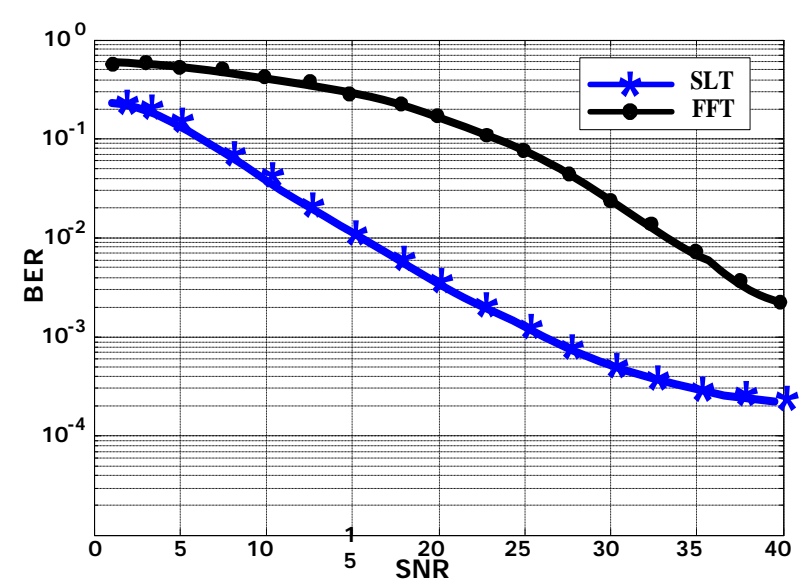

Fig. 9: Performance of SLT-MC-CDMA in FFC at maximum Doppler shift $=500 \mathrm{~Hz}$.

Three maximum Doppler shifts are used in the simulation: $5 \mathrm{~Hz}, 500 \mathrm{~Hz}$, and $1100 \mathrm{~Hz}$. The results of simulation are shown in Fig. 8, Fig. 9 and Fig. 10. It can be seen from the figures that BER performance of SLTMC-CDMA system is still better than the FFT-MCCDMA system. For example, Fig. 8 shows that SLTMC-CDMA has $B E R=10^{-3}$ at $S N R=25 \mathrm{~dB}$, while FFTMC-CDMA system has $B E R=10^{-3}$ at about $S N R=40 \mathrm{~dB}$. This is a $15 \mathrm{~dB}$ gain in SNR.

\subsection{Performance of proposed SLT-MC-CDMA system in Selective Fading Channel}

In Selective Fading Channel (SFC), the frequency complements of the transmitted signal are affected by uncorrelated changes, where the parameters of the channel correspond to two path system. For SFC many models are used in simulation and BER performance is compared for FFT-based and SLT-based MC-CDMA systems, the effect of path attenuation, delay and maximum Doppler shift of an echo was also taken in 
account. Fig. 11 shows the BER performance simulation of the SLT-MC-CDMA and the FFT-MC-CDMA systems for a SFC with path delay equal 1 sample, path gain equal $-8 \mathrm{~dB}$, and Doppler shift equal $5 \mathrm{~Hz}$. Fig. 12 and Fig. 13 plot the BER performance simulation of the systems for SFC with the same channel parameters except changing Doppler shift to $500 \mathrm{~Hz}$ and $1100 \mathrm{~Hz}$ respectively. It is seen that SLT-MC-CDMA system has better performance than FFT- MC-CDMA system. Fig. 14, Fig. 15, and Fig. 16 show the BER performance simulation of the SLT- MC-CDMA and FFT-MCCDMA systems for a SFC with path delay equal 1 sample, Doppler shift equal $5 \mathrm{~Hz}$, and path gain equal $1 \mathrm{~dB},-5 \mathrm{~dB}$ and $-12 \mathrm{~dB}$ respectively. It can be seen from plots that the performance of SLT-MC-CDMA is better than that of FFT-MC-CDMA for SNR less than $35 \mathrm{~dB}$, but for SNR greater than $35 \mathrm{~dB}$ FFT-MC-CDMA outperforms SLT-MC-CDMA system. Fig. 17, and Fig. 18 show the BER performance simulation of the SLTMC-CDMA and FFT-MC-CDMA system for a SFC with path gain equal $-5 \mathrm{~dB}$, Doppler shift equal $5 \mathrm{~Hz}$, and path delay equal 1 sample and 2 samples respectively. It can be seen from plots that BER performance of SLTMC-CDMA system is always better than that of FFTMC-CDMA system. For example from Fig. 17 SLTMC-CDMA has $B E R=10^{-3}$ at $S N R=21.5 \mathrm{~dB}$, while FFTMC-CDMA system has $B E R=10^{-3}$ at about $S N R=37.5 \mathrm{~dB}$. This is a $16 \mathrm{~dB}$ gain in SNR.

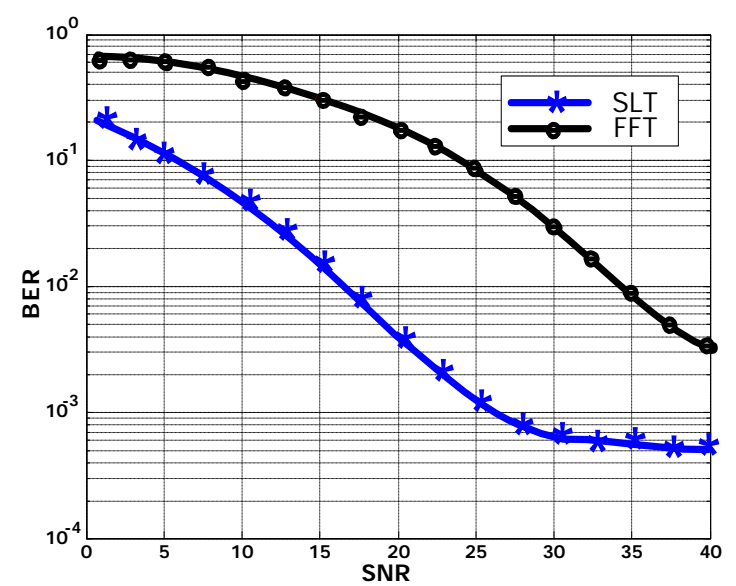

Fig. 10: Performance of SLT-MC-CDMA in FFC at max. Doppler shift $=1100 \mathrm{~Hz}$.

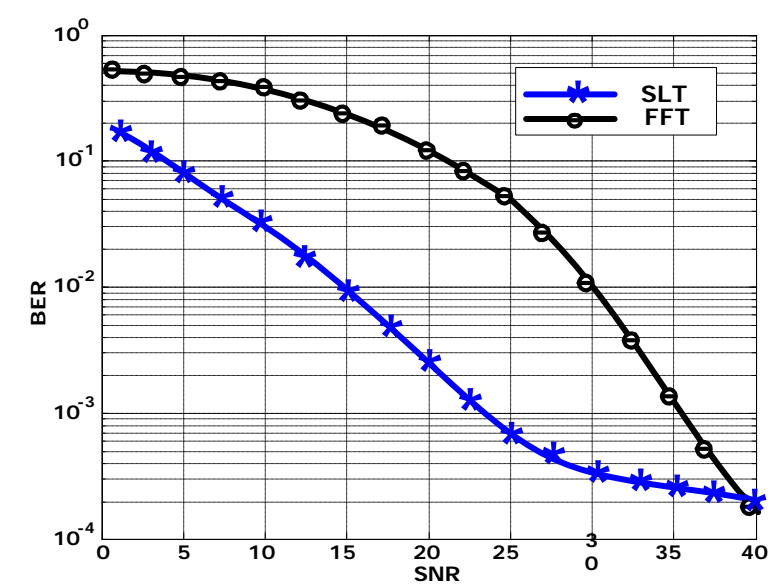

Fig. 11: Performance of SLT-MC-CDMA in SFC at maximum Doppler Shift $=5 \mathrm{~Hz}$.

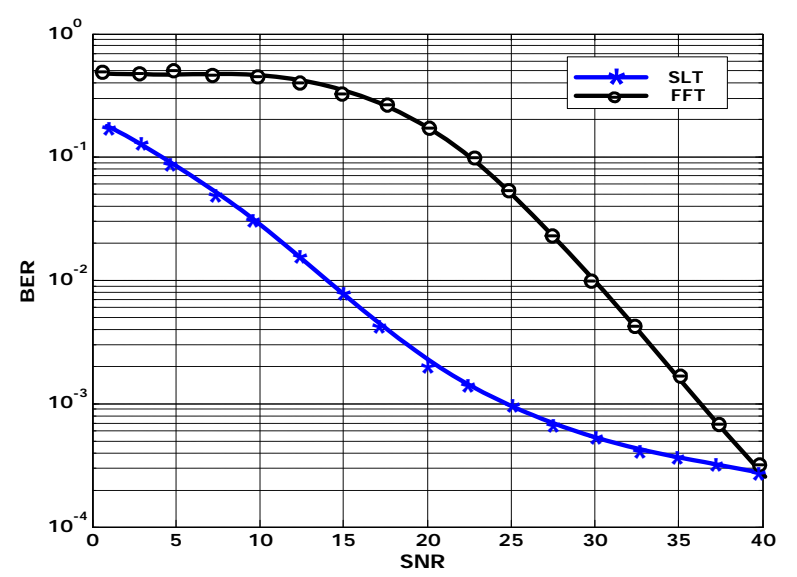

Fig. 12: Performance of SLT-MC-CDMA in SFC at maximum Doppler Shift $=500 \mathrm{~Hz}$.

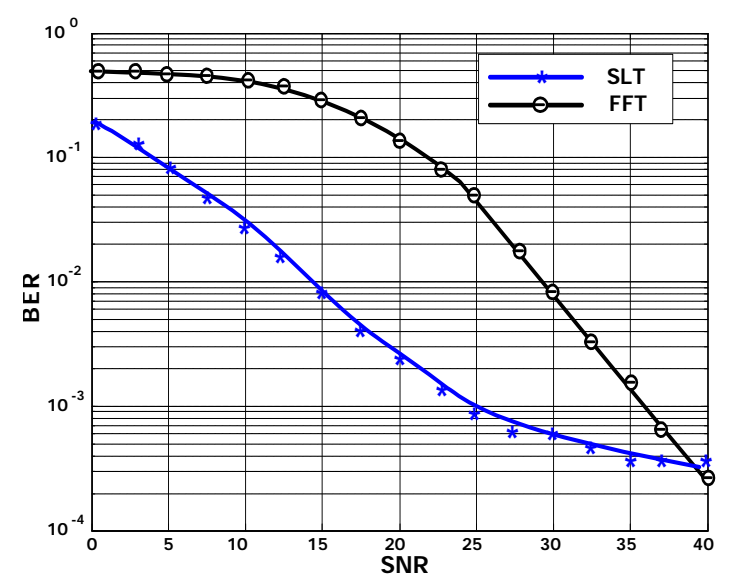

Fig. 13: Performance of SLT-MC-CDMA in SFC at max. Doppler Shift $=1100 \mathrm{~Hz}$. 


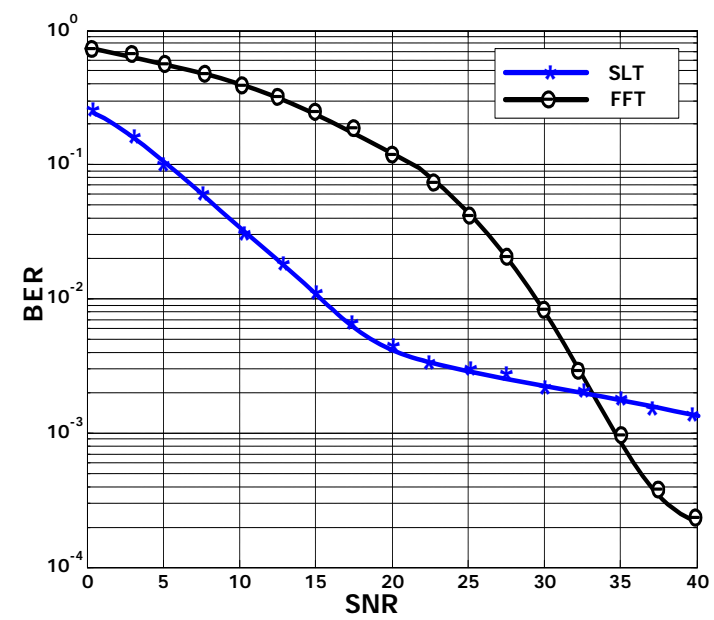

Fig. 14: Performance of SLT-MC-CDMA in SFC with path gain $=1 \mathrm{~dB}$.

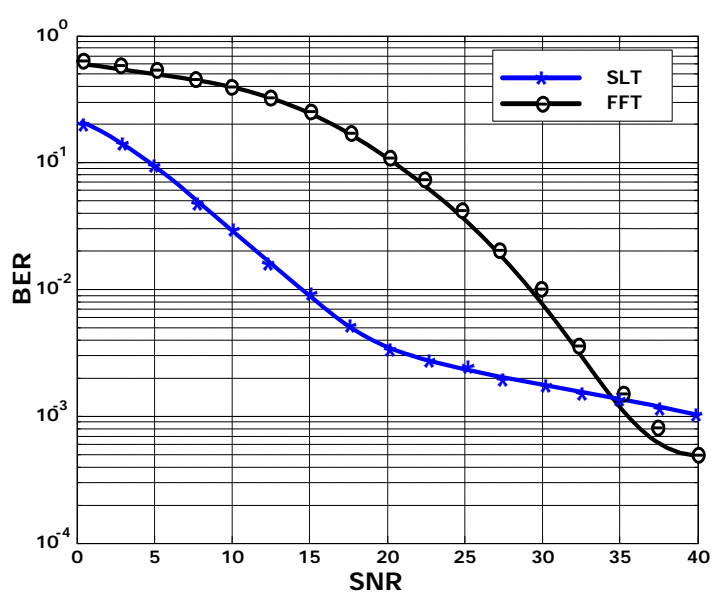

Fig. 15: Performance of SLT-MC-CDMA in SFC with path gain $=-5 \mathrm{~dB}$.

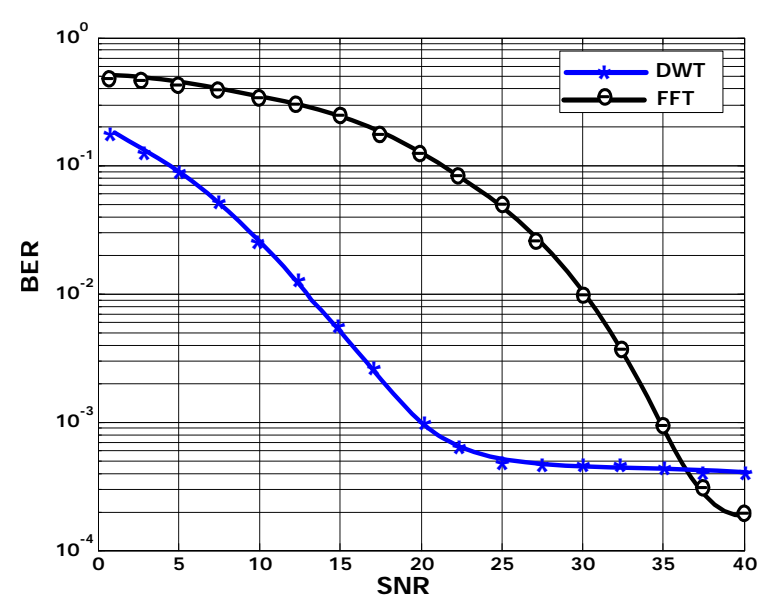

Fig. 16: Performance of SLT-MC-CDMA in SFC with path gain $=-12 \mathrm{~dB}$.

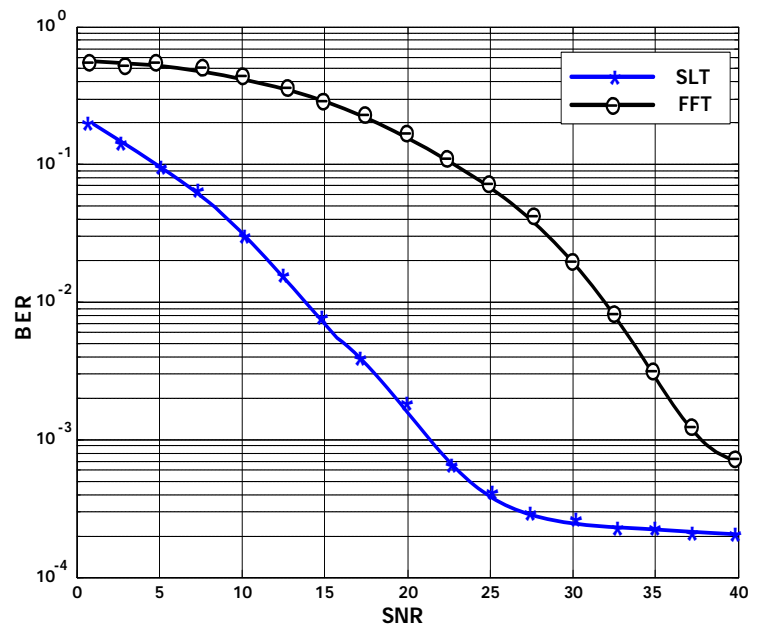

Fig. 17: Performance of SLT-MC-CDMA in SFC with path delay $=1$ sample.

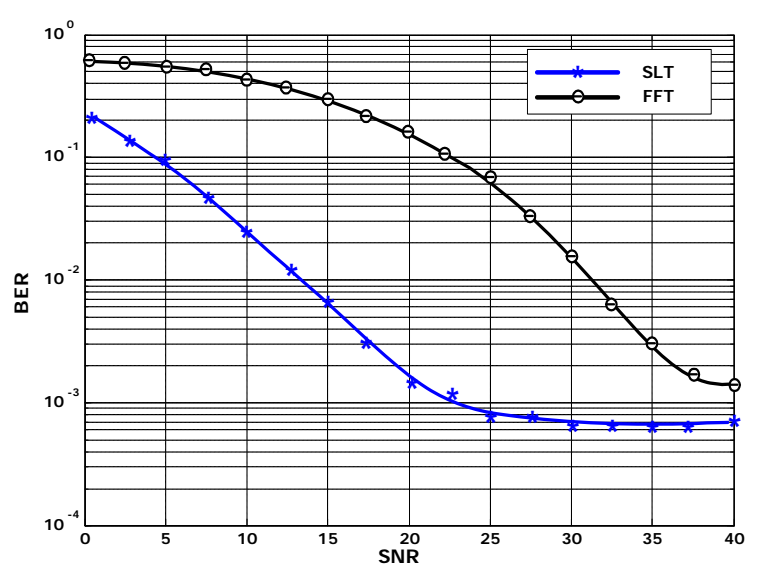

Fig. 18: Performance of SLT-MC-CDMA in SFC with path delay $=2$ sample.

\section{Conclusions}

In this paper a novel MC-CDMA system is designed and implemented, SLT-OFDM transceiver scheme is used in proposed MC-CDMA system instead of conventional FFT-OFDM. A robust to noise interference SLT is used to improve the performance of the communication system without a need to use the cyclic prefix.

Comparing the performance of SLT-MC-CDMA and FFT-MC-CDMA in AWGN channel, FFC, and SFC indicated that for AWGN channel SLT-MC-CDMA has performance gain about $15 \mathrm{~dB}$ compared with FFT-MCCDMA to achieve BER $=10^{-2}$. For FFC and for different maximum Doppler shift, SLT-MC-CDMA system has better performance than conventional system, it has performance gain of about $21 \mathrm{~dB}$ compared with FFTMC-CDMA to achieve BER $=10^{-2}$. In SFC proposed SLT-MC-CDMA has better performance than FFT-MCCDMA in all cases even when changing all channel 
parameters. Maximum Doppler shift badly affects the performance of SLT-MC-CDMA compared with other channel parameters. It can be noted that CDMA when combined with OFDM, has BER performance better than just using OFDM only. This can be addressed to spreading the original data stream and then modulating different carriers with each chip.

\section{References}

[1] Goldsmith A., Wireless Communications, Cambridge University Press, 2006.

[2] Ming K. and Chee T. "Hybrid OFDM-CDMA: A Comparison of MC/DS-CDMA, MC-CDMA and OFCDM," Dept of Electrical \& Electronic, Adelaide University, SA 5005, Australia. 2002.

[3] Ryu K. W., Park J. O. and Park Y. W., "Performance of multicarrier CS/CDMA in frequency-selective Rayleigh fading channels,” 2003 Spring IEEE 57th Semiannual Veh. Tech. Conf., vol. 2, pp. 1258-1262, April 2003.

[4] Nghi H. Tran, Nguyen Ha H., and Tho Le-Ngoc, "BitInterleaved Coded OFDM with Signal Space Diversity: Subcarrier Grouping and Rotation Matrix Design,” IEEE Transactions On Signal Processing, 55 (2007) pp. 11371149.

[5] Dasilva V. and Sousa E. S., "Multicarrier orthogonal CDMA codes for quasi synchronous communication systems," IEEE Jour. on selected area in Commun., vol. 12, no. 5, pp. 842-852, June 1994.

[6] Vandendorpe L., "Multitone spread spectrum multiple access communications system in a multipath rician fading channel," IEEE Trans. Veh. Technol., vol. 44, pp. 327-337, May 1995.

[7] Sourour E. A. and Nakagawa M., "Performance of orthogonal multicarrier CDMA in a multipath fading channel,” IEEE Trans. Commun., vol. 44, pp. 356-366, Mar. 1996.

[8] Shiro Kondo and Laurence B. Milstein, "Performance of multicarrier DS CDMA systems," IEEE Trans. on Communications, vol. 44, no. 2, Feb. 1996, pp. 238-246.

[9] Hara S. and Prasad R., "Overview of Multicarrier CDMA,” IEEE Commun. Magazine, vol. 35, no. 12, pp. 126-133, December 1997.

[10] Kattoush A. H., Waleed. A. M., Nihad S., "The Performance of Multiwavelets Based OFDM System Under Different Channel Conditions”, Elsevier Inc., Digital Signal Processing, Volume 20 Issue 2, March, 2010

[11] Yee N., JLinnartz-P., and Fettweis G., "Multicarrier CDMA in indoor wireless radio networks," Proc. of IEEE PIMRC'93, Yokohama, Japan, pp. 109-113, September 1993.

[12] Kaiser S. "On the Performance of Different Detection Techniques for OFDM-CDMA in fading channels," in IEEE ICC '95, June 1995, pp. 2059-2063.

[13] Chouly A., Brajal A. and Jourdan S., "Orthogonal multicarrier techniques applied to direct sequence spread spectrum CDMA systems," Proc. of IEEE GLOBECOM'93, Houston, USA, pp. 1723-1728, November 1993.

[14] Negash B. G. and Nikookar H. "Wavelet Based OFDM for Wireless Channels.”, International Research Center for Telecommunications-Transmission and Radar, Faculty of Information Technology and Systems, Delft University of Technology. 2001.

[15] Zhang H. et al, "Research of DFT-OFDM and DWTOFDM on Different Transmission Scenarios.", Proceedings of the $2^{\text {ed }}$ International Conference on Information Technology for Application (ICITA), 2004.

[16] Kattoush A. H. and Qasaymeh M. M. "Performance of a Slantlet Based OFDM Transceiver under Different Channel Conditions", IJITCS, vol.4, no.1, pp.64-72, 2012.

[17] Muayyadi A. and Abu-Rgheff M. A., "Wavelet-based multicarrier CDMA system and its corresponding multiuser detection,” IEE Proc. Commun., vol. 150, no. 6, pp. 445-452, December 2003.

[18] Haixia Zhang, Dongfeng Yuan, and Matthias Patzold, "Novel Study on PAPRs Reduction in Wavelet Based Multicarrier modulation Systems," Digital Signal Processing, vol. 17, no.1, pp. 272-279, Jan. 2007.

[19] Xiangbin Yu; Guangguo Bi; "Performance Analysis of Multicarrier CDMA System Based On Complex Wavelet Packet And Space-Time Block Codes In Rayleigh Fading Channel”, World Scientific Publishing, Journal of Circuits, Systems, and Computers, Vol. 15, No. 1 (2006) 57-74.

[20] Selesnick I. W., “The slantlet transform,” IEEE Trans. Signal Processing, 47 (1999), pp. 1304-1313.

[21] Alpert B., Beylkin G., Coifman R., and Rokhlin V., "Wavelet-like bases for the fast solution of second-kind integral equations”, SIAM J. Sci. Comput., 14 (1993), pp. 159-184.

[22] Daubechies I., "Ten Lectures on Wavelets", Philadelphia, PA: SIAM, 1992.

[23] Yew T. J., Multiwavelets and Scalable Video Compression, Ph.D. Thesis, Department of Electrical and Computer Engineering, National University of Singapore, 2002.

Abbas Hasan Kattoush received his M.S., and Ph.D. degrees in communication Eng. from former USSR in 1979 and 1984 respectively. For 10 years Dr Kattoush was a technical manager of a leading SAKHER computers company. He was a pioneer in computer networking and software engineering in Jordan. From 1993 to 2000 he worked at Applied Science University Amman Jordan where he was a founding member of the department of electrical and computer engineering. From 2000 to 2008 Dr. Kattoush was an associate professor at Electrical and Computer Engineering department at Al-Isra University, Amman-Jordan. Currently he is an associate professor at Tafila Technical University, Jordan. His areas of research interest include DSP, digital communication systems, phase unwrapping, filtering, and interpretation. He has authored several tenths of research articles, textbooks, and computer software systems.

Mahmoud Qasaymeh has done his Ph.D in Electrical Engineering / Telecommunications at Wichita State University (WSU), Kansas 2009. He did his MSc and B.Sc in Electrical Engineering / Telecommunications and Electronics at Jordan University of Science and Technology. Dr. Qasaymeh is currently with Tafila Technical University, Jordan. 